\title{
Stroke warning campaigns: delivering better patient outcomes? A systematic review
}

\author{
This article was published in the following Dove Press journal: \\ Patient Related Outcome Measures \\ 25 February 2015 \\ Number of times this article has been viewed
}

Lisa Mellon'

Frank Doyle'

Daniela Rohde'

David Williams ${ }^{2}$

Anne Hickey'

'Department of Psychology, Royal College of Surgeons in Ireland, Dublin, Ireland; ${ }^{2}$ Department of Geriatric and Stroke Medicine, Royal College of Surgeons in Ireland, Dublin, Ireland
Correspondence: Lisa Mellon Department of Psychology, Division of Population Health Sciences, Royal College of Surgeons in Ireland, I 23 St Stephens Green,

Dublin 2, Ireland

Tel +353 |402 2748

Fax $+353 \quad$ I402 2764

Email lisamellon@rcsi.ie
Background: Patient delay in presenting to hospital with stroke symptoms remains one of the major barriers to thrombolysis treatment, leading to its suboptimal use internationally. Educational interventions such as mass media campaigns and community initiatives aim to reduce patient delays by promoting the signs and symptoms of a stroke, but no consistent evidence exists to show that such interventions result in appropriate behavioral responses to stroke symptoms.

Methods: A systematic literature search and narrative synthesis were conducted to examine whether public educational interventions were successful in the reduction of patient delay to hospital presentation with stroke symptoms. Three databases, MEDLINE, CINAHL, and PsycINFO, were searched to identify quantitative studies with measurable behavioral end points, including time to hospital presentation, thrombolysis rates, ambulance use, and emergency department (ED) presentations with stroke.

Results: Fifteen studies met the inclusion criteria: one randomized controlled trial, two time series analyses, three controlled before and after studies, five uncontrolled before and after studies, two retrospective observational studies, and two prospective observational studies. Studies were heterogeneous in quality; thus, meta-analysis was not feasible. Thirteen studies examined prehospital delay, with ten studies reporting a significant reduction in delay times, with a varied magnitude of effect. Eight studies examined thrombolysis rates, with only three studies reporting a statistically significant increase in thrombolysis administration. Five studies examined ambulance usage, and four reported a statistically significant increase in ambulance transports following the intervention. Three studies examining ED presentations reported significantly increased ED presentations following intervention. Public educational interventions varied widely on type, duration, and content, with description of intervention development largely absent from studies, limiting the potential replication of successful interventions.

Conclusions: Positive intervention effects were reported in the majority of studies; however, methodological weaknesses evident in a number of studies limited the generalizability of the observed effects. Reporting of specific intervention design was suboptimal and impeded the identification of key intervention components for reducing patient delay. The parallel delivery of public and professional interventions further limited the identification of successful intervention components. A lack of studies of sound methodological quality using, at a minimum, a controlled before and after design was identified in this review, and thus studies incorporating a rigorous study design are required to strengthen the evidence for public interventions to reduce patient delay in stroke. The potential clinical benefits of public interventions are far-reaching, and the challenge remains in translating knowledge improvements and correct behavioral intentions to appropriate behavior when stroke occurs.

Keywords: acute stroke treatment, prehospital delay, onset to door times, public education, professional education, interventions 


\section{Introduction}

Thrombolysis with tissue plasminogen activator within a 3- to 4.5-hour window following onset of ischemic stroke is associated with improved patient outcome and reduction in disability, ${ }^{1}$ with the chances of a favorable outcome falling twofold for every 90-minute delay in treatment. ${ }^{2}$ Despite its proven efficacy, provision rates remain suboptimal internationally. ${ }^{3}$ Interventions targeted at the public aim to reduce patient delay in presentation with stroke symptoms, and thus play an important role in improving treatment rates for thrombolysis in ischemic stroke. Patient delay in accessing medical care has been cited as the biggest barrier to thrombolysis treatment for ischemic stroke, ${ }^{4-6}$ with prehospital delays estimated to account for up to $68 \%$ of total delay from onset to treatment. ${ }^{7}$ The aim of public-focused educational interventions is to reduce patient delay by educating the public of the common signs and symptoms of stroke and the correct course of action should symptoms occur.

Much of the published literature to date that examines the effectiveness of public educational interventions is concerned with nonbehavioral intervention end points where no definitive behavior occurs, such as stroke knowledge and intentions to call emergency medical services (EMS) ${ }^{8-11}$ However, the association between stroke knowledge and improved time to presentation is poor, ${ }^{12-14}$ and recent guidelines highlight that the only acceptable outcome of a health intervention is a behavioral outcome or a measurable consequence of the target behavior: ${ }^{15}$ ie, where the target behavior occurs following the intervention. Outcomes of a public stroke education intervention need to provide a measure of the clinical impact of the intervention. Measurable behavioral outcomes of an intervention, such as stroke onset to door (OTD) times, or measurable consequences such as thrombolysis rates, emergency department (ED) presentations with stroke symptoms, or ambulance dispatches help provide an estimate of the real effectiveness of an intervention of this type. The aim of this review was to examine the effectiveness of interventions that aim to reduce prehospital delay by systematically reviewing studies containing such behavioral end points.

\section{Methods}

\section{Search strategy}

Three electronic databases, MEDLINE, CINAHL, and PsycINFO, were searched from database start date to October 24, 2014 for educational interventions targeted at the public that aim to reduce prehospital delay and that had a measurable behavioral outcome. The key search term "stroke" was accompanied with all combinations of the terms "thromboly- sis", "tPA", “actilyse", “alteplase", "delay", "intervention", "education", "paramedic", "ambulance", "emergency", "awareness", "campaign", and "time". The Google Scholar search engine and reference lists for included papers were additionally searched in order to identify studies that may not have been identified through the database searches. Reference lists for identified review papers were scanned and crossreferenced with studies identified from the primary search. Two authors independently screened and reviewed abstracts and titles for relevant papers. Disagreements were discussed with a third reviewer.

\section{Inclusion and exclusion criteria}

Studies were included if a measurable behavioral outcome of a public educational intervention to reduce prehospital delay was assessed. Randomized controlled trials (RCTs), non-RCTs, and prospective, retrospective, and cross-sectional studies were included. Studies were excluded if the intervention outcome was stroke knowledge, attitudes, behavioral intentions, or any other nonbehavioral outcome. Studies were also excluded if the article was a review or discussion paper, if an English or German translation of the paper was not available, or if the article was not published in full in a peer-reviewed journal.

\section{Data extraction and synthesis}

The following data were extracted: author, publication year, location and setting, sample characteristics, intervention duration and content, behavioral outcomes assessed, and intervention results. Given the heterogeneity of study types, meta-analysis was not indicated, and a qualitative narrative synthesis was conducted.

\section{Outcomes}

The main outcomes of interest were reduction in OTD time, thrombolysis rates, activation of ambulance transports, ED presentations with stroke symptoms, and patient mortality.

\section{Quality assessment}

Critical appraisal of primary studies is an essential feature of systematic reviews; however, no consensus exists on the "gold standard" appraisal method for assessing methodological quality. ${ }^{16}$ The Crowe Critical Appraisal Tool (CCAT) ${ }^{17}$ was selected for the purposes of this review. It is a generic critical appraisal tool that has been reported to be simple to implement and applicable to all research designs in health, with obtained scores being directly comparable. Studies are scored from 0 to 5 on eight dimensions: preamble, 
introduction, design, sampling, data collection, ethical matters, results, and discussion. Each dimension includes up to three subcategories with a number of descriptors marked as present, absent, or not applicable. The total score, ranging from 0 to 40, may be displayed as a percentage. The CCAT demonstrates good reliability and ${ }^{18,19}$ construct validity, ${ }^{20}$ comprehensively assesses the key aspects of research, and can be utilized across different research designs.

\section{Results}

Database searches identified 4,561 citations, with 3,006 remaining after duplicate removal. Three studies were additionally identified through Google Scholar searches. Fifteen studies met the inclusion criteria. Figure 1 outlines the screening process and the reasons for study exclusion.

Table 1 displays the main characteristics of the final studies. A single cluster RCT ${ }^{21}$ was identified, and two time series design studies ${ }^{22,23}$ were identified. Three studies ${ }^{24-26}$ were controlled before and after studies, and five studies ${ }^{27-31}$ were uncontrolled before and after studies. There were two retrospective observational studies ${ }^{32,33}$ and two prospective observational studies. ${ }^{34,35}$ The main outcome of interest, reduction in prehospital delay, was assessed in 13 of the studies. ${ }^{21,23-31,34,35}$ Rates of thrombolysis were assessed in eight studies. ${ }^{21,22,24,26,29,31,33,34}$ Five studies ${ }^{23,25,29,30,32}$ examined ambulance dispatches for stroke, three studies ${ }^{22,23,28}$ examined ED presentations, and two studies ${ }^{21,26}$ examined patient mortality as behavioral indicants of intervention effectiveness.

Study quality was assessed independently by two reviewers using the CCAT, with disagreements discussed with a third reviewer. All papers were rated on the eight dimensions of the CCAT, and a total score for each study was derived from the average score of the independent ratings and expressed as a percentage. Overall, the methodological quality was mixed. As only one study was identified as an RCT design, the majority of studies lacked quality in research design, management of bias, and sampling. Data collection

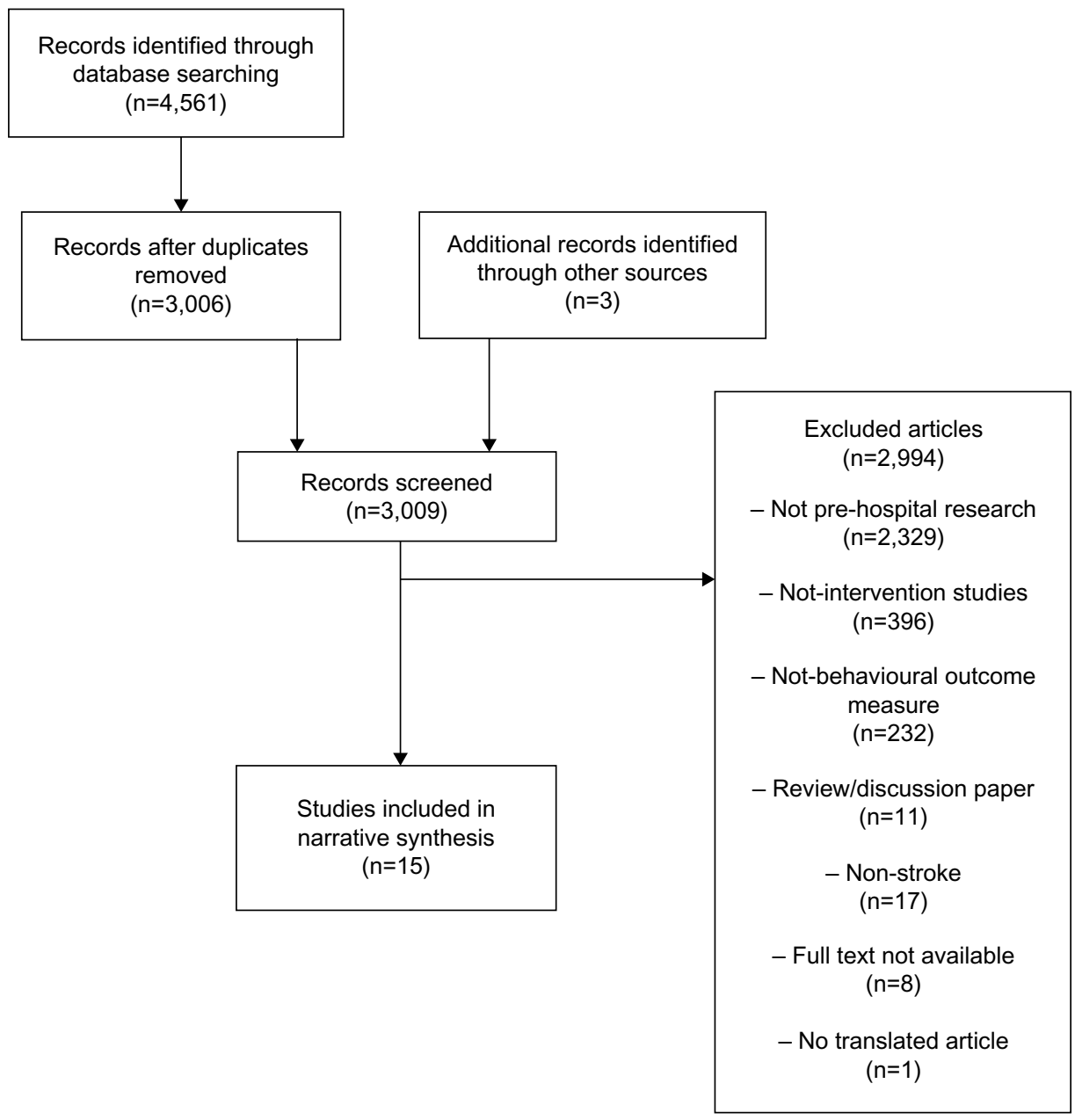

Figure I Flow chart of studies screened, excluded (with reasons), and included in the review. 


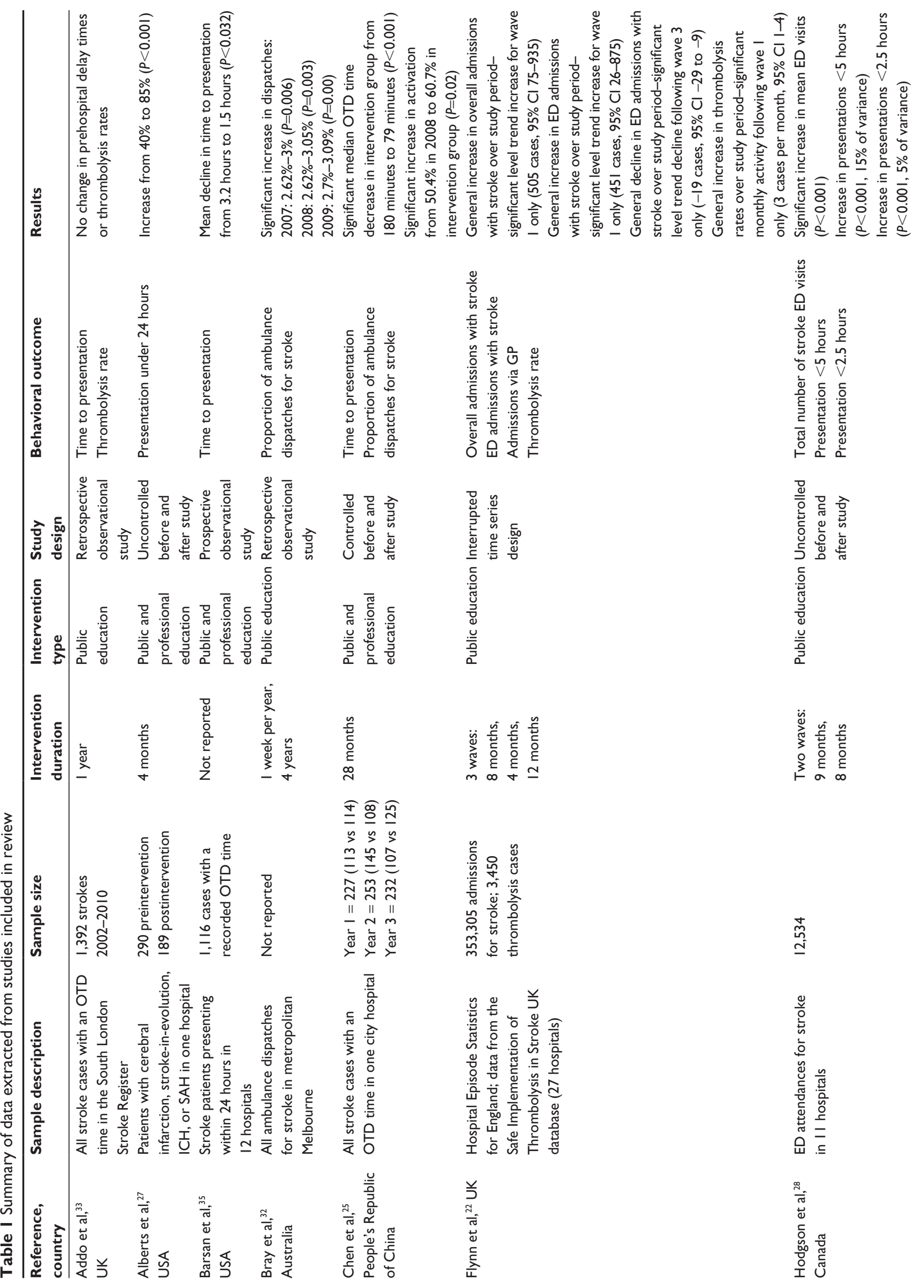




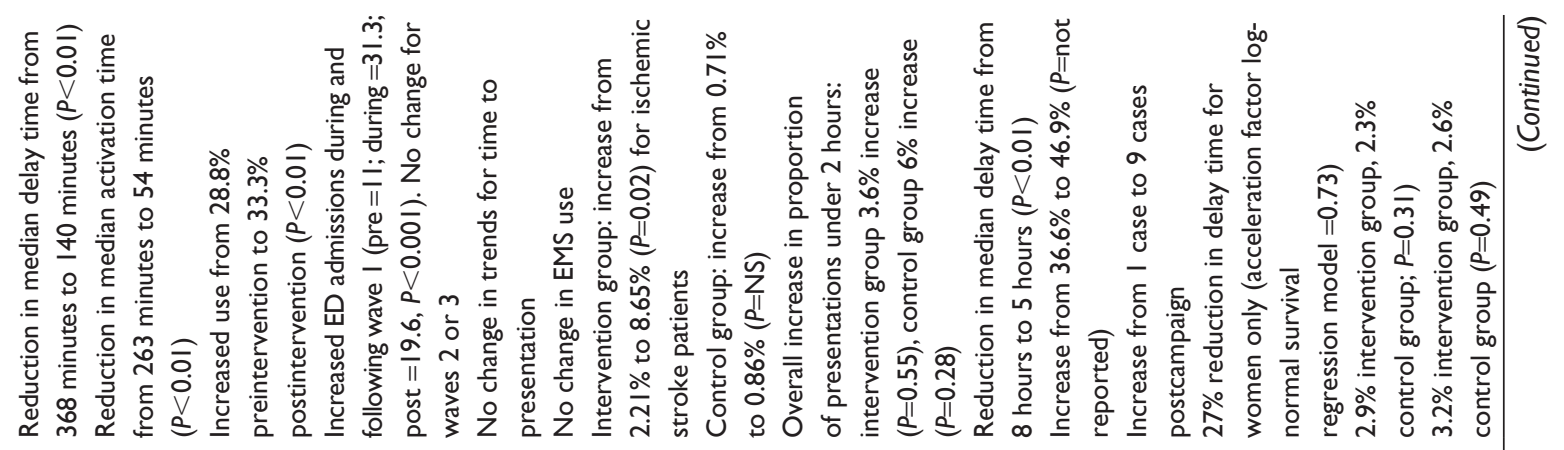
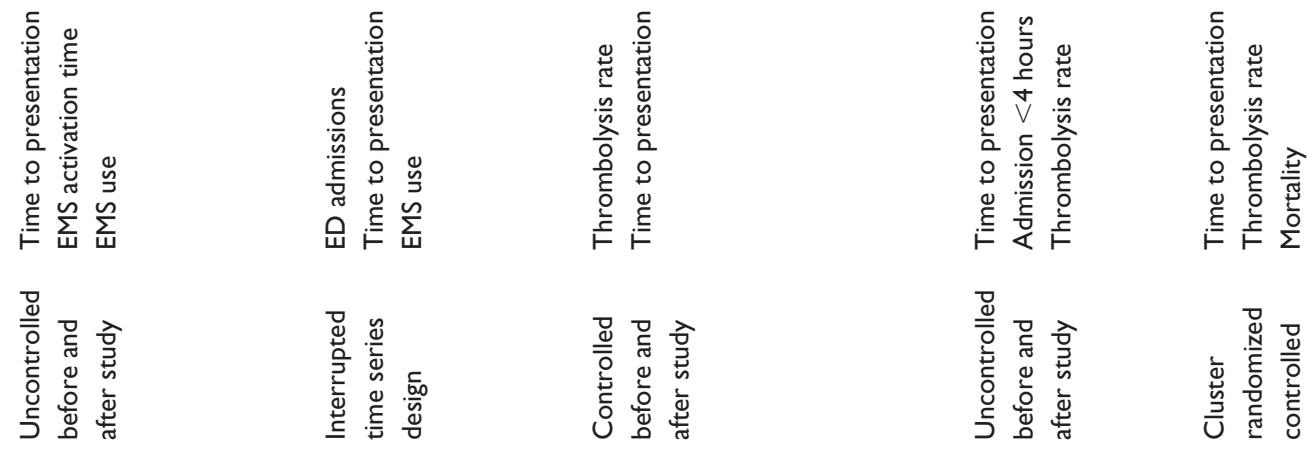

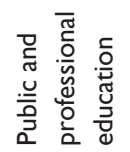

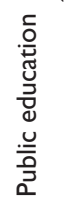

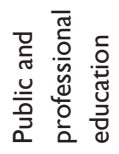

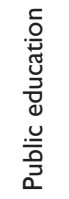
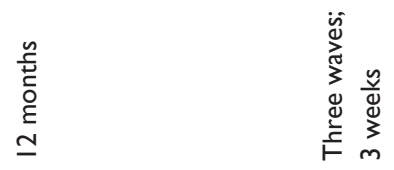

$\stackrel{n}{5}$
$\stackrel{5}{0}$
$\stackrel{0}{E}$
$\underline{-}$

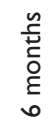
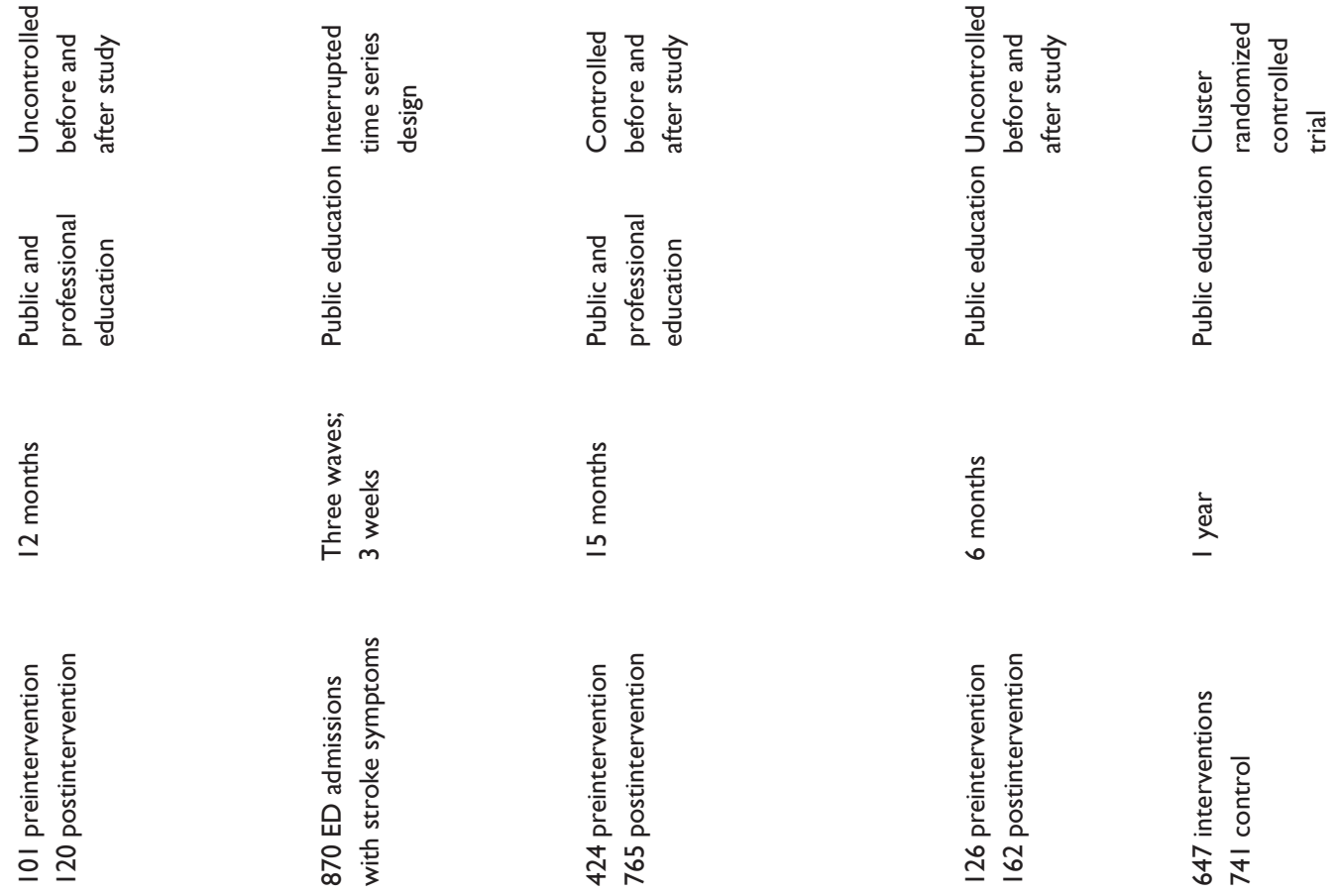

$\stackrel{\text { ळ }}{\stackrel{1}{ \pm}}$
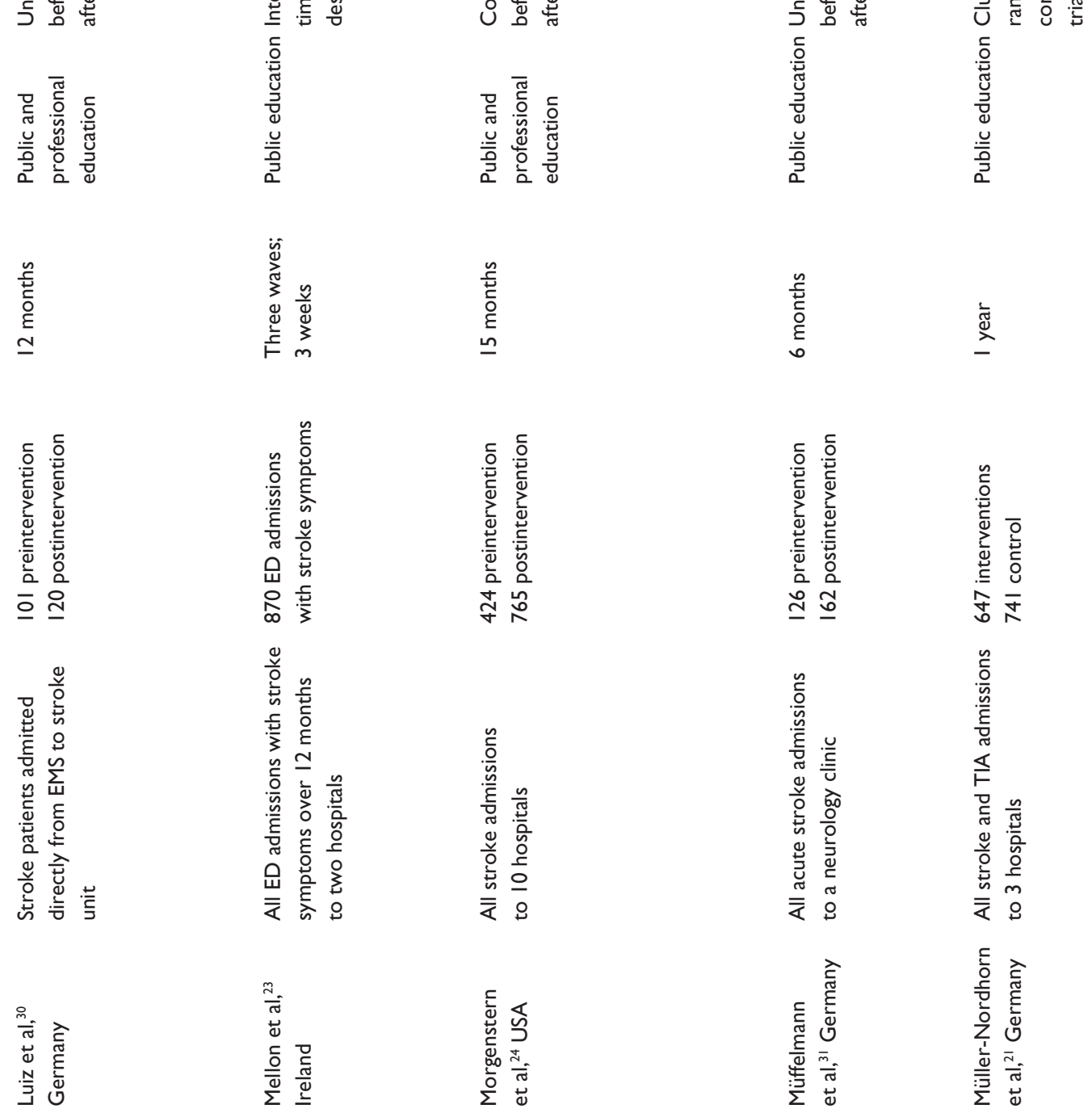


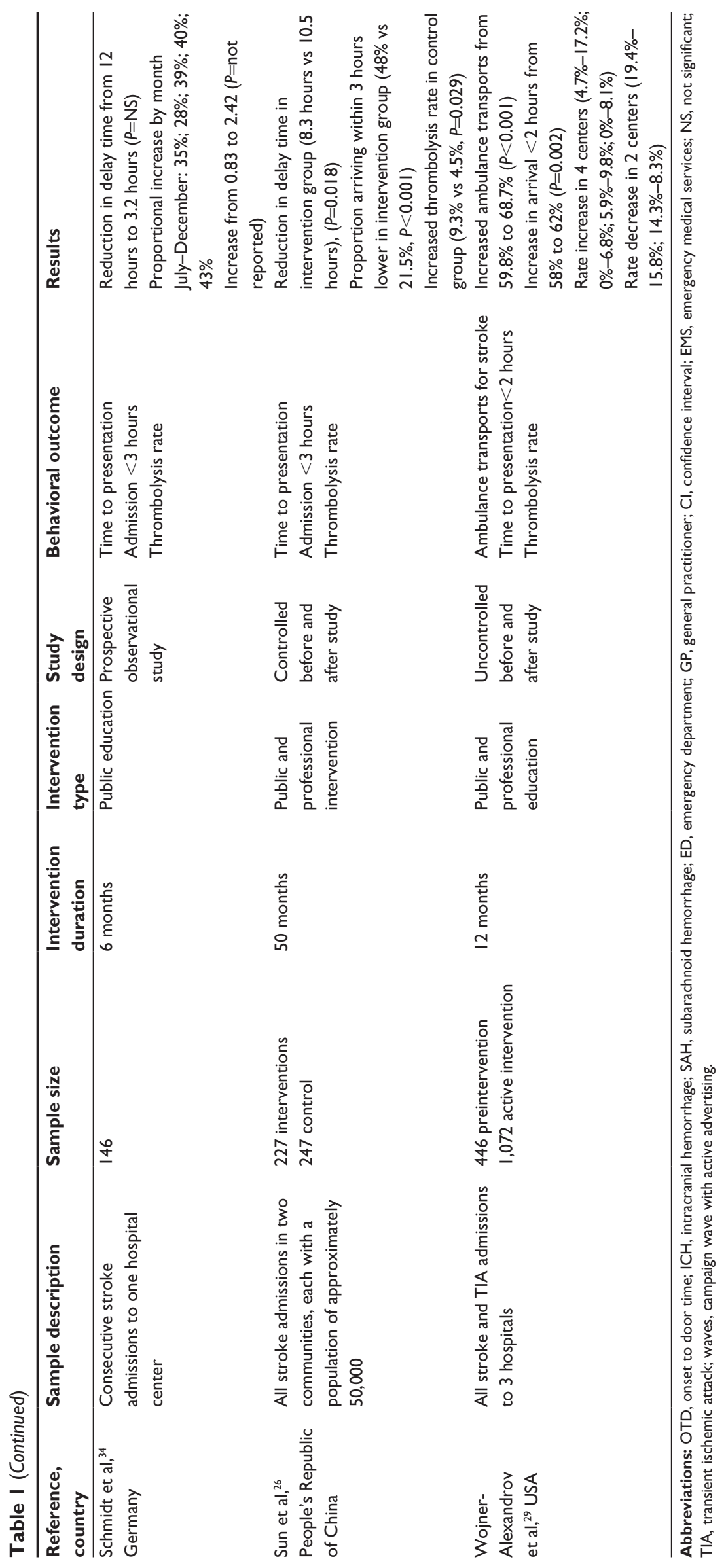


methods and comprehensive reporting of results were also identified as poor quality for some studies.

\section{Study population}

Data collection settings were the hospital ED in the majority of cases, with information obtained mainly from patient medical records or hospital stroke registries. One study ${ }^{35}$ obtained additional data from the patient and/or family, with another study ${ }^{32}$ retrospectively examining patients with an ambulance dispatch for stroke in an urban ambulance service over a 10-year period. In three studies, ${ }^{27,31,35}$ patients were recruited as part of a thrombolysis trial.

\section{Intervention characteristics}

Intervention content

Table 2 summarizes the content of the interventions described in the included studies. Intervention descriptions varied considerably between studies. Five studies described mass media campaigns aimed at the general public, all using a specific slogan to promote stroke awareness. The Australian, ${ }^{32} \mathrm{UK},{ }^{22,33,36}$ and $\mathrm{Irish}^{23}$ studies described the FAST (Face, Arm, Speech, Time) campaign, which highlights three main symptoms of stroke and the need to contact EMS immediately. The Canadian study ${ }^{28}$ utilized the SUDDENS campaign, highlighting 'sudden' onset of weakness, trouble speaking, vision, headache, and/or dizziness. The remaining studies described both public and professional education campaigns that were broadcast at a local and regional level. The professional campaigns focused on reducing prehospital and in-hospital delays, from initiation of EMS contact to treatment in the acute hospital setting. These were aimed at professionals involved in acute stroke care, including paramedics, ED staff, and general practitioners. Three studies ${ }^{24,26,27}$ used educational messages that described thrombolysis treatment, emphasizing the time-dependent nature of the treatment in addition to its efficacy. The $\mathrm{RCT}^{21}$ posted educational packs to inhabitants aged over 50 years in 48 randomized zip codes, each containing a personalized letter outlining signs and symptoms of stroke, a bookmark, and a sticker outlining the main symptoms and EMS contact details.

\section{Intervention development}

Only the intervention by Morgenstern et $\mathrm{al}^{24}$ comprehensively described the stages of development of the intervention. The community intervention component involved role-modeling of the appropriate response to stroke symptoms by publicly advertising stroke survivors in the community and their successful experience of thrombolysis. The purpose of community role-modeling was to demonstrate that immediate stroke response was associated with better outcome. Morgenstern et $\mathrm{al}^{24}$ also developed a local advisory board and conducted a telephone survey $(n=656)$ for the purposes of identifying factors related to intention to react rapidly to signs of stroke. The authors additionally conducted focus groups with stroke survivors to assess factors that contributed to delay in time to hospital presentation with stroke. The professional education component involved systems change in hospitals, change of perceived norms in the medical community, and reinforcement of behavior change.

\section{Patient delay times}

Thirteen studies examined change in patient prehospital delay times as an outcome following a public educational intervention. The majority of studies reported statistically significant effects in terms of reducing patient delay, with ten studies reporting a statistically significant reduction in prehospital delay following the intervention, with the magnitude of change varying per study.

\section{RCT study}

The $\mathrm{RCT}^{21}$ reported a statistically significant reduction of $27 \%$ in prehospital delay in women in the intervention group, with no change observed in men.

\section{Controlled before and after studies}

The controlled study by Morgenstern et $\mathrm{al}^{24}$ involving community and professional intervention in the US reported an overall decrease in prehospital delay; however, the decrease was general and not specific to the intervention group. Hence, the public intervention was not associated with this overall decrease. Two Chinese controlled before and after studies reported significant reductions in patient delay. The study by Chen et $\mathrm{al}^{25}$ evidenced a significant yearly decline in median OTD time from 280 minutes in 2008 to 79 minutes in 2010 in the intervention community, with no changes reported in the controlled community. Sun et $\mathrm{al}^{26}$ reported a reduction of 2.2 hours in the intervention group for OTD time, with a significantly greater proportion $(26 \%)$ in the intervention group arriving to hospital in less than 3 hours.

\section{Time series study}

An Irish time series analysis by Mellon et $\mathrm{al}^{23}$ reported no effect of the FAST campaign on OTD time over a 12-month period. 


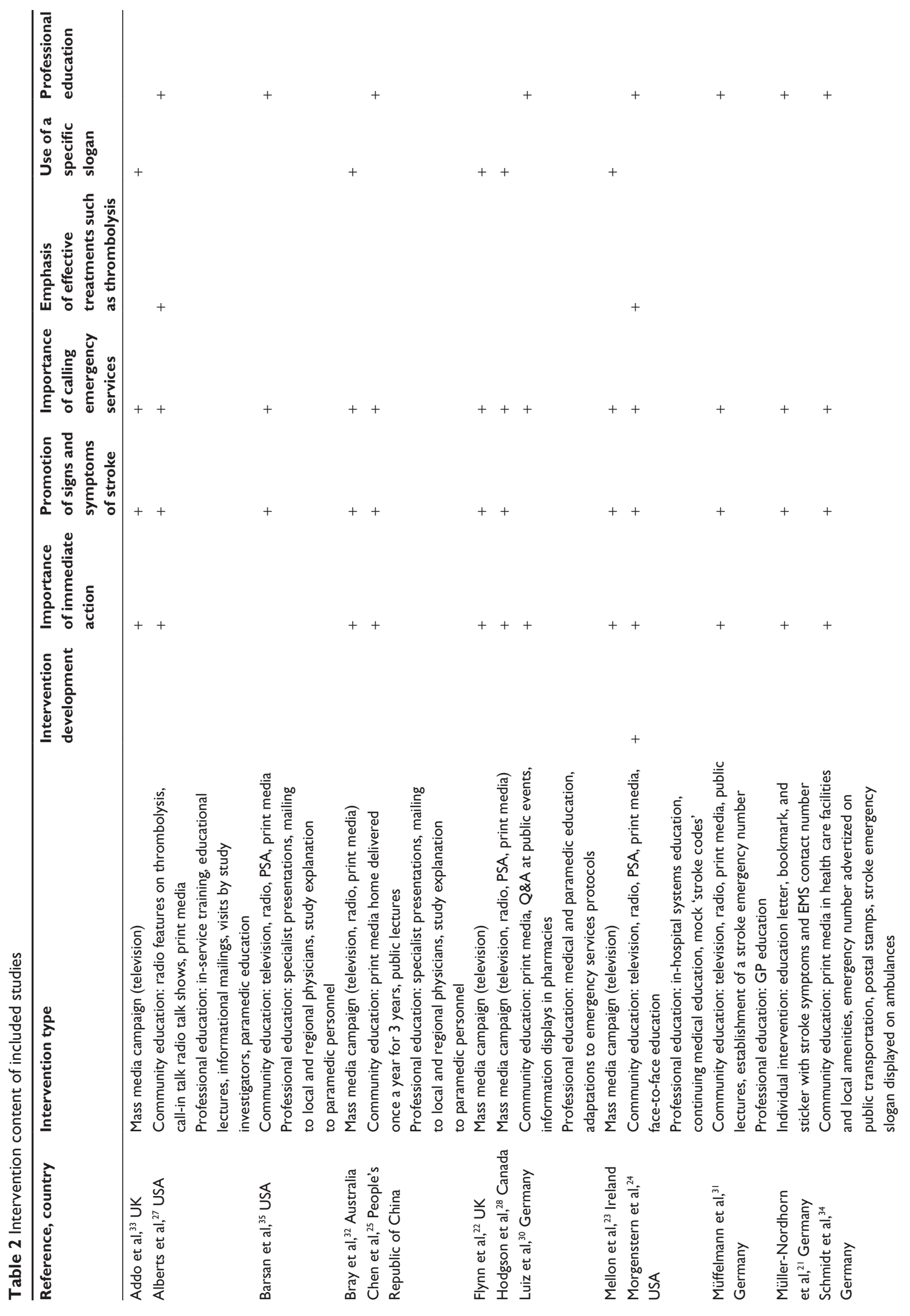




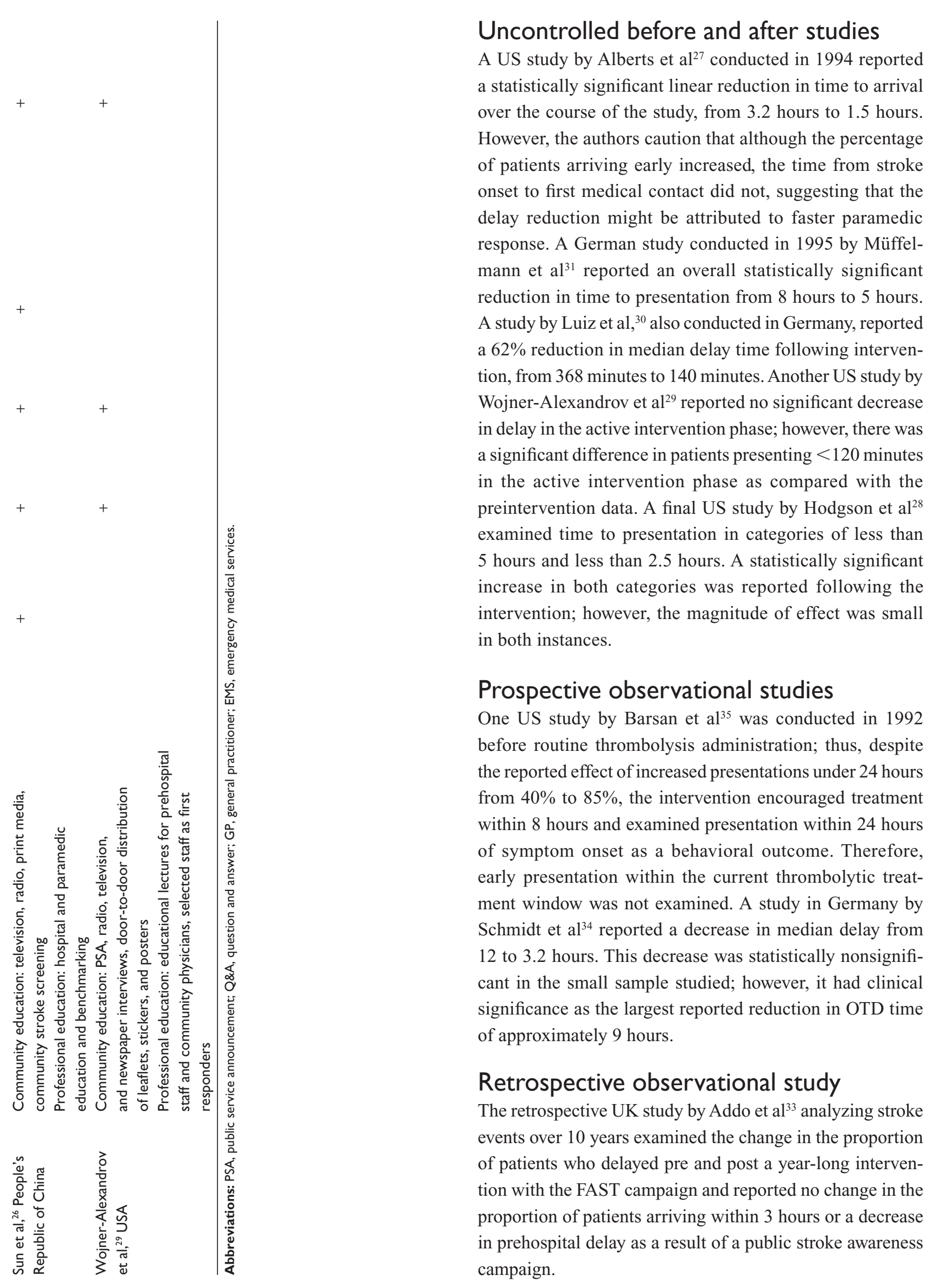




\section{Increase in thrombolysis rates}

Eight studies examined thrombolysis rates as a behavioral outcome measure, with only three studies reporting a statistically significant increase in thrombolysis rates following a public educational intervention.

Interestingly, the $\mathrm{RCT}^{21}$ reported no significant difference in thrombolysis rates between the intervention and control groups. The time series analysis by Flynn et $\mathrm{al}^{22}$ analyzed data from the Safe Implementation of Thrombolysis in Stroke UK database, which included data from 27 hospital sites across England. Findings showed an overall increase in thrombolysis rates over the study period, with specific increases observed following waves 1 and 3 of the FAST campaign. The controlled before and after study by Moregenstern et $\mathrm{al}^{24}$ reported a statistically significant increase in thrombolysis rates from $2.21 \%$ to $8.65 \%$ for ischemic stroke in the intervention community following an educational intervention, with no change observed in the control community. A follow-up study ${ }^{36}$ within this population reported a sustained increase in thrombolysis rates $(11.2 \%)$ in the intervention community at 6 months postintervention. The controlled before and after study by Sun et $\mathrm{al}^{26}$ reported a $4.8 \%$ increase in thrombolysis rates in the intervention community following a community-based intervention. An uncontrolled before and after study by Wojner-Alexandrov et $\mathrm{al}^{29}$ examined the change in thrombolysis rates in nine treatment centers from the preintervention to the active intervention phase. No consistent trend was observed over the intervention phase, although four hospitals reported a rise in thrombolysis (only one of these being a statistically significant rise), while two hospitals in this cohort reported decreased thrombolysis rates. Another uncontrolled before and after study by Müffelmann et $\mathrm{al}^{31}$ reported an increase in thrombolysis cases, from one case to nine cases, following a public education campaign. The prospective study by Schmidt et $\mathrm{al}^{34}$ reported an overall increase in thrombolysis rates but did not provide statistical analysis of the reported change. The retrospective study by Addo et $\mathrm{al}^{33}$ reported no significant difference in thrombolysis rates before or after a public stroke awareness campaign.

\section{Activation of ambulance transport}

Ambulance transports for stroke were outcome measures in five studies, with four reporting statistically significant increases in ambulance transportations for stroke following an educational intervention. The controlled study by Chen et $\mathrm{al}^{25}$ reported that the percentage of patients calling EMS increased per year from 2008 , with a $10.8 \%$ overall increase noted and an increased proportion evident in the intervention community in 2010 . The study by Bray et al ${ }^{32}$ examined the impact of a multimedia educational intervention on the proportional increase in ambulance dispatches over a 10-year period. The authors reported a yearly significant increase in ambulance dispatches in the month after the annual stroke awareness week from 2007 once the message to call an ambulance in response to stroke was added to the existing media message. The study by Wojner-Alexandrov et $\mathrm{al}^{29}$ reported a significant increase in paramedic transports for stroke during the intervention phase as compared with the preintervention phase. The retrospective study by Luiz et $\mathrm{al}^{30}$ reported a $4.5 \%$ increase in EMS transports for stroke postintervention. The same study also examined the time taken from symptom onset to EMS activation (ie, calling the EMS telephone number) and found a significant reduction of $79 \%$ in median activation time to 54 minutes.

\section{ED presentations}

Three studies examined ED presentations with stroke as an outcome measure, with all studies reporting some statistically significant effects, with increases in ED presentations for stroke observed. One before and after study by Hodgson et $\mathrm{al}^{28}$ examined ED presentations with stroke symptoms and reported a mean increase in ED presentations over the study period. The authors reported that exposure to the mass media campaign accounted for $9 \%$ of the total variance in ED admissions for stroke, $15 \%$ of the variance for presentations under 5 hours, and $5 \%$ of the variance for presentations within 2.5 hours. The UK time series analysis by Flynn et al ${ }^{22}$ of all ED activity for England from May 2007 to February $2011^{22}$ revealed that there was a general increase in ED presentations over the study period. However, when ED trends immediately following the FAST campaign were examined, only the time period following wave 1 of the FAST campaign evidenced a significant increase. The second time series analysis by Mellon et $\mathrm{al}^{23}$ reported similar findings to the UK analyses. The Irish data examined ED presentations with stroke symptoms over a 12-month period, with significant increases in ED presentations only observed following wave 1 of the FAST campaign.

\section{Mortality}

The $\mathrm{RCT}^{21}$ examined the percentage of deaths during hospital stay between the intervention and control groups, reporting no statistical difference in mortality between the groups. A controlled before and after study by Sun et $\mathrm{al}^{26}$ reported no difference in the 90-day fatality rate between the intervention and control groups; however, interestingly, the intervention 
group had significantly higher functional outcome scores, measured on the Barthel Index, than the control group at 90 days poststroke.

\section{Discussion}

This review examined the effectiveness of public interventions aimed at reducing patient prehospital delay in acute ischemic stroke. Outcomes of interest were focused on patient behavior (patient delay, EMS use, and ED presentation) or measurable consequences of behavior (thrombolysis rates and mortality). The findings suggest that educational interventions aimed at the public have some positive effects on reducing patient delay to hospital presentation and other behavioral indicants such as ambulance use, ED presentation with suspected stroke, and thrombolysis rates. However, results must be interpreted with caution, as large heterogeneity was evident between study designs and methodology.

Interventions such as multimedia campaigns have demonstrated efficacy in increasing stroke knowledge and awareness and the intention to respond to stroke as an emergency. ${ }^{37-39}$ However, bridging the gap between behavioral intention and behavior itself remains a challenge in acute stroke care and has similarly been identified as a key challenge in other health contexts, such as acute myocardial infarction..$^{40}$ The intervention in this review that evidenced the largest and, more crucially, more sustained behavioral change in thrombolysis rates involved a scientifically based, multifaceted behavioral intervention that was developed from focus groups with stroke survivors in order to identify themes and issues that contribute to delay. ${ }^{24}$ The theory-based strategy involved role-modeling of response to stroke by promoting community figures who were stroke survivors and demonstrated that immediate response to stroke symptoms results in a better outcome. Furthermore, this type of intervention did more than just demonstrate signs and symptoms to the public; it outlined the time-dependent nature of successful treatments for ischemic stroke, which highlights this strategy as a possible key component in interventions to reduce delay in presentation with stroke symptoms.

The intervention in the RCT study ${ }^{21}$ did not have an observable impact on delay behavior. This finding has been similarly reported in RCT studies examining the effect of interventions to reduce delay in presentation with symptoms of an acute myocardial infarction. Stroke researchers can potentially learn from the more established acute myocardial infarction literature in order to improve treatment outcomes for acute ischemic stroke. Both conditions are life-threatening events with acute onset and similar mechanism of infarction. Successful treatment involves revascularization, and hence both have similar need for time-dependent intervention. A large randomized clinical trial ${ }^{41}$ to reduce prehospital delay in acute coronary syndrome $(n=5,322)$ found that a one-to-one educational and counseling intervention with patients with documented heart disease at 2-year follow-up resulted in no change to prehospital delay times, although the experimental group was more likely to call emergency services and self-medicate with aspirin following symptom onset. Secondary analysis of this cohort ${ }^{42}$ aimed to characterize the patients who best responded to the intervention, in order to understand the mechanisms by which interventions work. It was found that decreasing anxiety was associated with prehospital delay reduction, and authors suggest that tailoring interventions to target psychosocial and emotional responses to acute onset of symptoms may be more effective than addressing knowledge, attitudes, or intention. Methods such as community role-modeling of stroke survivors may be useful in reducing potential anxiety and removing potential psychosocial barriers to timely acute stroke care.

Seven studies in this review that reported significant effects described interventions that targeted both professional and public behavior. Professional education may have contributed to improved thrombolysis rates through improved prehospital paramedic response and in-hospital systemic delay reductions. This interaction effect was not examined in any study presented here, and it is therefore difficult to draw conclusions on which element (public or professional) was most effective. Patient recognition and action in the wake of stroke symptom onset is the first step in the symptom delay trajectory, and the studies included in this review focused on public interventions that targeted this step in the onset to treatment delay trajectory. The next two stages, from initiation of medical contact to hospital arrival, and from arrival to treatment, are out of the patient's control and rely on prompt professional action to reduce delays to treatment. Significant advances in prehospital management of stroke by paramedic protocols and EMS systems, coupled with in-hospital improvements in the management of stoke, have translated into shorter overall onset to treatment times. For example, a recent single-center study ${ }^{43}$ reported a door-to-needle time of 20 minutes, which was attributed to concurrent in-hospital systemic changes and the employment of a prehospital notification system, which resulted in a smoother transition from prehospital to in-hospital emergency care. A multicenter intervention $^{44}$ also reported a clinically significant increase in thrombolysis use in the target population following a 
multicomponent intervention, although this increase was not statistically significant. A shorter door-to-needle time affords the stroke patient slightly more time to recognize and respond accordingly to stroke symptoms without risk of missing the thrombolysis treatment window. The findings from the time series analyses included in this review provide further evidence for this gradual improvement in pre and in-hospital acute management of stroke. Flynn et $\mathrm{al}^{22}$ highlight that in the study period from 2007 to 2011, there were significant increases in ED presentations for stroke, coupled with a decline in time-consuming general practitionerreferred stroke cases, and an increase in thrombolysis rates over time. These service improvements occurred in parallel with improvements in patient response attributable to the FAST campaign. Changing the public perception of acute stroke is a challenging and lengthy process involving large, heterogeneous populations. Perhaps a sustainable reduction in onset-to-needle time may be best achieved over a shorter time frame by improving in-hospital acute stroke care, as in-hospital interventions have the advantage of focusing on a single network or organization in a controlled environment, such as the ED or EMS network.

This review had some limitations. The heterogeneity of the included studies limited the comparability of the interventions, and critical appraisal of the included studies revealed that potential bias or confounders were not successfully addressed in the majority of studies. Only one RCT was identified, and therefore methodological deficiencies of included studies limit the weight that can be given to the demonstrated effects. Some of the included studies also retrospectively examined intervention effects as a secondary analysis to a larger study, which again decreases the methodological rigor. Studies in this area should utilize, at a minimum, a controlled before and after design or interrupted time series design if an RCT design is not feasible, ${ }^{42}$ in order to associate any observed changes with the specified intervention.

\section{Conclusions}

In conclusion, this review found that interventions to educate the public on the correct response to stroke symptoms evidenced varying degrees of success, with most interventions successful in initiating correct public responses when stroke occurred. Studies that were particularly successful in achieving reductions in prehospital delay adopted a combined multilevel approach to education, incorporating mass media, targeted community education, and professional education. Sustained reduction in patient delay and increased thrombolysis administration were achieved by an intervention that specifically promoted the use of thrombolysis for stroke and its associated benefits. There is a need for well-designed research trials with clearly described intervention components to provide evidence for the effectiveness of interventions to reduce delayed presentation with stroke symptoms.

\section{Acknowledgment}

This project was funded by the Health Research Board in Ireland through the HRB PhD Scholars Programme in Health Services Research, Grant No PhD/2007/16.

\section{Disclosure}

The authors report no conflicts of interest in this work.

\section{References}

1. Hacke W, Kaste M, Bluhmki E, et al. Thrombolysis with alteplase 3 to 4.5 hours after acute ischaemic stroke. NEMJ. 2008;359:1317-1329.

2. Lees KR, Bluhmki E, von Kummer R, et al. Time to treatment with intravenous alteplase and outcome in stroke: an updated pooled analysis of ECASS, ATLANTIS, NINDS, and EPITHET trials. Lancet. 2010;375:1695-1703

3. Schumacher HC, Bateman BT, Boden-Albala B, et al. Use of thrombolysis in acute ischaemic stroke: analysis of the Nationwide Inpatient Sample 1999-2004. Ann Emerg Med. 2007;50:99-107.

4. Evenson KR, Rosamond WD, Morris DL. Prehospital and in-hospital delays in acute stroke care. Neuroepidemiology. 2001;20:65-76.

5. Evenson KR, Foraker RE, Rosamond WD. A comprehensive review of prehospital and in-hospital delay times in acute stroke care. Int $J$ Stroke. 2009;4:187-199.

6. Kwan J HP, Sandercock P. A systematic review of barriers to delivery of thrombolysis for acute stroke. Age Ageing. 2004;33:116-121.

7. Wester P, Radberg J, Lundgren B, Peltonen M. Factors associated with delayed admission to hospital and in-hospital delays in acute stroke and TIA: a prospective, multicenter study. Stroke. 1999;30(1):40-48.

8. Silver F, Rubini F, Black D, Hodgson C. Advertising strategies to increase public awareness of the warning signs of stroke. Stroke. 2003;34:1965-1969.

9. Reeves MJ, Hogan JG, Rafferty AP. Knowledge of stroke risk factors and warning signs among Michigan adults. Neurology. 2002;59: $1547-1552$.

10. Reeves MJ, Rafferty AP, Aranha AA, Theisen V. Changes in knowledge of stroke risk factors and warning signs among Michigan adults. Cerebrovasc Dis. 2008;25:385-391.

11. Fogle C, Oser C, Troutman P, et al. Public education strategies to increase awareness of stroke warning signs and the need to call 911. J Public Health Manag Pract. 2008;14:E17-E22.

12. Fussman C, Rafferty AP, Lyon-Callo S, Morgenstern LB, Reeves MJ. Lack of association between stroke symptoms knowledge and intent to call 911. Stroke. 2010;41:1501-1507.

13. Carroll C, Hobart J, Fox C, Teare L, Gibson J. Stroke in Devon: knowledge was good, but action was poor. J Neurol Neurosurg Psychiatry. 2004;75:567-571.

14. Reeves MJ. Reducing the delay between stroke onset and hospital arrival: is it an achievable goal? J Am Heart Assoc. 2012;1(3):e002477.

15. Michie S, Johnston M. Theories and techniques of behaviour change. Developing a cumulative science of behaviour change. Health Psychol Rev. 2012;6:1-6.

16. Moja LP, Telaro E, D’Amico R, Moschetti I, Coe L, Liberati A. Assessment of methodological quality of primary studies by systematic reviews: results of the metaquality cross sectional study. BMJ. 2005; 330:1053. 
17. Crowe M, Sheppard L. A review of critical appraisal tools show they lack rigor: alternative tool structure is proposed. J Clin Epidemiol. 2011;64:79-89.

18. Crowe M, Sheppard L, Campbell A. Reliability analysis for a proposed critical appraisal tool demonstrated value for diverse research designs. J Clin Epidemiol. 2012;65:375-383.

19. Crowe M, Sheppard L, Campbell A. Comparison of the effects of using the Crowe Critical Appraisal Tool versus informal appraisal in assessing health research: a randomised trial. Int J Evid Based Healthc. 2011;9:444-449.

20. Crowe M, Sheppard L. A general critical appraisal tool: an evaluation of construct validity. Int J Nurs Stud. 2011;48:1505-1516.

21. Müller-Nordhorn J, Wegscheider K, Nolte C, et al. Population-based intervention to reduce prehospital delays in patiens with cerebrovascular events. Arch Intern Med. 2009;169:1484-1490.

22. Flynn D, Ford G, Rodgers H, Price C, Steen N, Thomson RG. A time series evaluation of the FAST national stroke awareness campaign in England. PLoS One. 2014;9:e104289.

23. Mellon L, Hickey A, Doyle F, Dolan E, Williams D. Can a media campaign change health service use in a population with stroke symptoms? Examination of the first Irish stroke awareness campaign. Emerg Med J. Epub July 26, 2013.

24. Morgenstern LB, Staub L, Chan W, et al. Improving delivery of acute stroke therapy: the TLL Temple Foundation Stroke Project. Stroke. 2002;33:160-166.

25. Chen S, Sun H, Zhao X, et al. Effects of comprehensive education protocol in decreasing pre-hospital stroke delay among Chinese urban community population. Neurol Res. 2013;35(5):522-528.

26. Sun $X$, Zhang N, Wang T, et al. Public and professional education on urgent therapy for acute ischaemic stroke: a community-based intervention in Changsha. Neurol Sci. 2013;34:2131-2135.

27. Alberts MJ, Perry A, Dawson DV, Bertels C. Effects of public and professional education on reducing the delay in presentation and referral of stroke patients. Stroke. 1992;23:352-356

28. Hodgson C, Lindsay P, Rubini F. Can mass media influence emergency department visits for stroke? Stroke. 2007;38:2115-2122.

29. Wojner-Alexandrov AW, Alexandrov AV, Rodriguez D, Persse D, Grotta JC. Houston Paramedic and Emergency Stroke Treatment and Outcomes Study (HoPSTO). Stroke. 2005;36:1512-1518.

30. Luiz T, Moosmann A, Koch C, Behrens S, Daffertshofer M, Ellinger K. [Optimized logistics in the prehospital management of acute stroke]. Anasthesiol Intensivmed Notfallmed Schmerzther. 2001;36:735-741. German.

31. Müffelmann B, Glahn J, Kunz S, Busse O. [Strategies for early admission of stroke patients]. Nervenarzt. 1995;66:111-115. German.
32. Bray JE, Mosley I, Bailey M, Barger B, Bladin C. Stroke public awareness campaigns have increased ambulance dispatches for stroke in Melbourne, Australia. Stroke. 2011;42:2154-2157.

33. Addo J, Ayis S, Leon J, Rudd AG, McKevitt C, Wolfe C. Delay in presentation after an acute stroke in a multiethnic population in South London: the South London Stroke Register. J Am Heart Assoc. 2012;1(3):e001685.

34. Schmidt NK, Huwel J, Weisner B. Causes of a prolonged prehospital phase in patients admitted to a stroke unit. Can it be influenced by campaigns to educate the public? Nervenarzt. 2005;76:181-185.

35. Barsan W, Brott T, Broderick J, Haley E, Levy DE, Marler J. Urgent therapy for acute stroke. Effects of a stroke trial on untreated patients. Stroke. 1994;25:2132-2137.

36. Morgenstern LB, Bartholomew K, Grotta J, Staub L, King M, Chan W. Sustained benefit of a community and professional intervention to increase acute stroke therapy. Arch Intern Med. 2003;163. 2198-2202.

37. Wall HK, Beagan BM, O’Neill J, Foell KM, Boddie-Willis CL. Addressing stroke signs and symptoms through public education: the Stroke Heros Act FAST campaign. Prev Chronic Dis. 2008;5:A49.

38. Schneider AT, Pancioli AM, Khoury JC, et al. Trends in community knowledge of the warning signs and risk factors for stroke. JAMA 2003;289:343-346.

39. Miyamatsu N, Kimura K, Okamura T, Iguchi Y, Nakayama H, Toyota A, et al. Effects of public education by television on knowledge of early stroke symptoms among a Japanese population aged 40 to 74 : years a controlled study. Stroke. 2012;43:545-549.

40. Moser DK, Kimble LP, Alberts MJ, et al. Reducing delay in seeking treatment by patients with acute coronary syndrome and stroke: a scientific statement from the American Heart Association Council on Cardiovascular Nursing and Stroke Council. J Cardiovasc Nurs. 2007; 22:326-343

41. Dracup K, McKinley S, Riegel B, Moser DK, Meischke H, Doering LV. A randomised clinical trial to reduce patient prehospital delay to treatment in acute coronary syndrome. Circ Cardiovasc Qual Outcomes. 2009;2:524-532.

42. Riegel B, Elmi A, Moser DK, et al. Who listens to our advice? A secondary analysis of data from a clinical trial testing an intervention designed to decrease delay in seeking treatment for acute coronary syndrome. Patient Educ Couns. 2011;85:e33-e38.

43. Meretoja A, Strbian D, Mustanoja S, Tatlisumak T, Lindsberg PJ, Kaste M. Reducing in-hospital delay to 20 minutes in stroke thrombolysis. Neurology. 2012;79(4):306-313.

44. Dirks M, Niessen LW, van Wijngaarden JD, et al. Promoting thrombolysis in acute ischemic stroke. Stroke. 2011;42(5):1325-1330.
Patient Related Outcome Measures

\section{Publish your work in this journal}

Patient Related Outcome Measures is an international, peer-reviewed, open access journal focusing on treatment outcomes specifically relevant to patients. All aspects of patient care are addressed within the journal and practitioners from all disciplines are invited to submit their work as well as healthcare researchers and patient support groups.

\section{Dovepress}

The manuscript management system is completely online and includes a very quick and fair peer-review system. Visit http://www.dovepress. com/testimonials.php to read real quotes from published authors. 